\title{
Transdermal Nicotine Patch Effects on EEG Power Spectra and Heart Rate Variability During Sleep of Healthy Male Adults
}

\author{
Jong-Bae Choi' ${ }^{1}$, Yu-Jin G. Lee ${ }^{2}$, and Do-Un Jeong ${ }^{3 凶}$ \\ ${ }^{1}$ Department of Psychiatry, Yongin Mental Hospital, Yongin, Republic of Korea \\ ${ }^{2}$ Department of Psychiatry, Seoul Metropolitan Hospital, Seoul, Republic of Korea \\ ${ }^{3}$ Department of Psychiatry, Seoul National University Hospital, Seoul, Republic of Korea
}

\begin{abstract}
Objective The effect of transdermal nicotine patch on sleep physiology is not well established. The current study aimed to examine the influence of nicotine patch on homeostatic sleep propensity and autonomic nervous system.

Methods We studied 16 non-smoking young healthy volunteers with nocturnal polysomnography in a double blind crossover design between sleep with and without nicotine patch. We compared the sleep variables, sleep EEG power spectra, and heart rate variability.

Results The night with nicotine patch showed significant increase in sleep latency, wake after sleep onset, and stage 1 sleep; and decrease in total sleep time, sleep efficiency, and percentage of REM sleep. Also, spectral analysis of the sleep EEG in the night with nicotine patch revealed decreased slow wave activity in stage 2 and REM sleep and increased alpha activity in the first NREM-REM sleep cycle. Heart rate variability showed no differences between the 2 nights, but the low to high ratio (a parameter indicative of sympathetic nervous system activity) positively correlated with wake after sleep onset in night with nicotine patch.

Conclusion Transdermal nicotine patch significantly disrupts sleep continuity, sleep architecture, and homeostatic sleep propensity. The overactivation of the sympathetic nervous system may be responsible for these changes. Psychiatry Investig 2017;14(4):499-505
\end{abstract}

Key Words Nicotine, Sleep, Polysomnography, Spectrum analysis, Heart rate variability.

\section{INTRODUCTION}

The role of nicotine in human sleep is not well defined. Some evidence suggests nicotine has sedating effects at low doses and alerting effects at high doses. ${ }^{1}$ A prior study found that smokers had prolonged sleep latency compared with nonsmokers and that acute abstinence normalized sleep. ${ }^{2}$ In studies using transdermal nicotine patches, Davila et al. ${ }^{3}$ reported that transdermal nicotine decreased total sleep time (TST), sleep efficiency, and REM sleep time and prolonged initial sleep latency (SL) in non-smoking and obese sleep disordered breathing patients. Gilin et al. ${ }^{4}$ suggested that transdermal nicotine was associated with early morning awakening and reduced total REM sleep time in a dose-dependent fashion. Sa-

Received: January 18, 2017 Revised: March 13, 2107

Accepted: April 18, 2017 Available online: June 16, 2017

$\triangle$ Correspondence: Do-Un Jeong, MD, PhD, Professor Emeritus

Department of Psychiatry, Seoul National University Hospital, 101 Daehak-ro, Jongno-gu, Seoul 03080, Republic of Korea

Tel: +82-2-2072-2457, Fax: +82-2-744-7241, E-mail: jeongdu@snu.ac.kr

(a) This is an Open Access article distributed under the terms of the Creative Commons Attribution Non-Commercial License (http://creativecommons.org/licenses/by$\mathrm{nc} / 4.0$ ) which permits unrestricted non-commercial use, distribution, and reproduction in any medium, provided the original work is properly cited.
lin-Pasucual et al. ${ }^{5}$ also reported that transdermal nicotine caused sleep fragmentation and reduction of REM sleep time in non-smoking volunteers.

So far, most of the studies on the role of nicotine were based on classical sleep variables calculated from visual sleep stage scoring. ${ }^{2-7}$ While visual sleep scoring is useful for characterization of sleep architecture and continuity, it is insufficient for more refined, quantitative analysis of sleep electroencephalogram (EEG). ${ }^{8}$ All-night spectral analysis of the sleep EEG provides quantitative information on the continuously fluctuating patterns of the EEG during sleep.9 A study comparing the EEG spectral power as well as the polysomnographic findings of smokers and non-smokers, found lower percentage of delta activity and higher percentage of alpha activity in the early part of sleep. ${ }^{10}$

Delta activity which is also referred to "slow wave activity (SWA)" encompasses components of the EEG signal in the frequency range of approximately 0.5 to $4.5 \mathrm{~Hz}$ as obtained by spectral analysis. SWA represents a physiological marker of the homeostatic facet of sleep regulation, as it increases with wake duration, peaks in early sleep, and declines in late sleep. ${ }^{11-13}$ The decline in SWA during sleep can be approximated by an 
exponential decay and its rise in the course of waking by a saturating exponential function. ${ }^{11}$ This is evidenced by the physiological enhancement of "sleep pressure" from sleep deprivation which enhances power density in the entire delta and theta frequency range $(0.5-10 \mathrm{~Hz})$, whereas power in the frequency band of sleep spindles $(12-15 \mathrm{~Hz})$ is reduced. ${ }^{14-17}$ About the mechanisms responsible for the increase of SWA after prolonged sleep deprivation, Tononi and Cirelli ${ }^{18}$ suggested that the increase in SWA occurs because during wakefulness, many cortical circuits undergo synaptic potentiation, as evidenced by the widespread induction of long-term potentiation-related genes in the brain of awake animals.

Specific frequency bands in EKG spectral analysis are suggested to be related to parasympathetic or sympathetic nervous system activity. ${ }^{19}$ Relative power in high frequency (HF) areas, usually from 0.15 to $0.5 \mathrm{~Hz}$, may refer to parasympathetic nervous system activity. Lower frequencies (LF) from 0.05 to $0.15 \mathrm{~Hz}$ are characteristic of both parasympathetic and sympathetic influences. Because the LF power is a combination of parasympathetic and sympathetic effects, investigators frequently infer sympathetic nervous system activity from the ratio of low (parasympathetic and sympathetic) to high (predominantly parasympathetic) (LHR) power. Nicotine is reported to increase arousal and attention and elevate heart rate and blood pressure $e^{20-22}$ by changes in the sympathetic activity. Past studies have tried to explore the underlying autonomic mechanism of transdermal nicotine effects on human sleep by EKG spectral analysis. ${ }^{23-26}$

In the current study, in order to examine nicotine effects on homeostatic sleep propensity and autonomic nervous system function, we analyzed both sleep EEG and EKG power spectra in the nights transdermal nicotine patch compared to the nights with placebo patch. We predicted that transdermal nicotine would disrupt human sleep architecture, suppress homeostatic sleep propensity, and facilitate sympathetic nervous system activity.

\section{METHODS}

\section{Subjects and study protocol}

Sixteen nonsmoking young healthy male adult volunteers (24 \pm 1.15 years, range: $22-26)$ were studied with polysomnography. Inclusion criteria were as the following: 1) nonsmoker males, 2) no history of mental and physical disorders at present and in the past that might influence sleep architecture. Depression was ruled out by using Hamilton Rating Scale for Depression ${ }^{27}$ and Beck Depression Inventory ${ }^{28}$ (cut-off score of 10), 3) no heavy snoring or obesity (body mass index $>28), 4$ ) no sleep disorders such as sleep apnea syndrome, idiopathic hypersomnia, narcolepsy, and periodic limb move- ment disorder, confirmed with overnight polysomnography, 5) no history of drug abuse, and 6) no use of medications that may affect sleep. The study was explained to them. Then, a signed consent approved by the Institutional Review Board in Yongin Mental Hospital was obtained. The subjects were asked to abstain from ethanol and caffeine for at least 3 days prior to each sleep recording and during the experiment, Breath-ethanol concentrations were measured with a Lion Alcometer S-D2 (Lion Laboratories plc., Barry, Wales, UK).

Polysomnography was conducted for three nights. The first night was for adaptation and to rule out sleep disorders. On the second night, one transdermal nicotine or placebo patch was administered in a double blind randomized crossover design. One nicotine patch contained $14 \mathrm{mg}$ of nicotine. Both nicotine and placebo patches were generously provided by Sam Yang Pharmaceutical Company as the identical shape and color. Every patch was applied at 18:00 on the upper outer arm about 4-5 hours before retiring to bed. Recording started between 22:00 and 23:00. The patch was removed the next morning. After a washout period of minimum 84 hours, the third night polysomnography was carried out and the placebo or nicotine patch was administered in a counter-balanced order. Subjects were allowed to maintain their usual lifestyle except during experimental night times.

\section{Polysomnographic recordings and digitization}

Polysomnographic recordings were done with Grass model 78 polysomnograph, which included electroencephalography (EEG), left and right electro-oculography (EOG), submentalis electromyography (EMG), left and right anterior tibialis EMG, electrocardiography (ECG), snoring microphone, oronasal airflow, chest and abdominal respiratory movements, and arterial oxygen saturation.

Two channels of EEG were recorded at $\mathrm{C} 3 / \mathrm{A} 2$ and $\mathrm{O} 2 / \mathrm{A} 1$ according to the international $10-20$ system. ${ }^{29}$ EOG electrode placement was done following the standard method. ECG electrode was attached at modified lead II position. The snoring microphone was attached at the surface area nearest to the vocal cord. Oro-nasal airflow was monitored with the thermocouple positioned at the mouth and the nostrils. Chest and abdominal breathing movements were monitored with impedance pneumograph applied to the chest and abdomen at the maximum excursion of breathing. Oxymeter probe was attached at the tip of the non-dominant second finger. All recordings were done at the paper speed of $10 \mathrm{~mm} / \mathrm{second}$. Behavioral monitoring was also done with infrared closed circuit camera system.

Polysomnography data were digitized and analyzed by the digital polysomnography system, developed jointly by Division of Sleep Studies in Seoul National University Hospital and 
Institute of Biomedical Engineering in Seoul National University using visual $\mathrm{C}++$ programming language (Microsoft visual $\mathrm{C}++$, version 6.0, USA) and a high performance analogto digital (AD) and digital signal processing (DSP) card based on a transputer in order to record and process simultaneously in real time. EEG and EKG data were sampled with a frequency of $250 \mathrm{~Hz}$.

\section{Visual sleep scoring and sleep variable definition}

Sleep stages were scored visually in 30-second epochs from the recording, according to Rechtschaffen and Kales criteria. ${ }^{30}$ Movement time epochs were not scored separately but were included in the wake stage.

All-night sleep variables were derived from the visual scoring of recordings using standard criteria and were grouped into 2 catogories: sleep continuity measures and sleep architecture measures. Sleep continuity measures included time in bed (TIB), TST, sleep efficiency in percent (TST/TIB $\times 100)$, sleep latency (time from lights-out to the first occurrence of a stage 1 or 2 epoch), and REM sleep latency (time from sleep-onset to the first epoch of stage REM). Sleep architecture measures included duration and percentage of time spent asleep in the different stages of sleep (stage 1, 2, 3, 4, REM, and slow wave sleep, i.e. the sum of sleep stages 3 and 4 ).

\section{Power spectral analysis of sleep EEG and EKG data}

For each subject, one EEG derivation (C3-A2) was selected to determine the power spectra. The spectral analysis was performed with the fast Fourier transform (FFT) algorithm. The epoch length was 2 seconds (256 points), and truncating error was reduced with applying Hanning window. In addition, the values for 15 adjacent 2 -second epochs were averaged to yield power density values for 30 -second periods. Thus, sleep visual scores of each 30-second epoch were synchronized with power density values. The values of adjacent $0.5-\mathrm{Hz}$ frequency bins were collapsed into slow wave activity (0.5-4.5 Hz; SWA), spindle frequency activity $(12-15 \mathrm{~Hz}$; SFA), theta $(4-7.5 \mathrm{~Hz})$, alpha $(8-12.5 \mathrm{~Hz})$, beta1 $(13-21.5 \mathrm{~Hz})$, and beta2 $(22-30 \mathrm{~Hz})$ frequency bands. Power spectral parameters were calculated within a specific period of time. Consecutive NREM-REM sleep cycles were defined according to modified criteria of Feinberg and Floyd ${ }^{31}$ by the succession of an NREM sleep episode of at least 15 minutes' duration and an REM sleep episode of at least 5 minutes' duration.

For each subject, 300 continuous beats of EKGs without artifact were chosen from both stage 2 and REM sleeps and were also used for power spectral analysis. We compared the heart rate variability including $L F$ and HF power, LHR, very low frequency power (VLF), R-R means, and R-R variance between the nights with and without transdermal nicotine patch.

\section{Statistical analyses}

Statistical software package SPSS 11.0 for windows was used. Tests for normality of continuous measures were made with the Kolmogorov-Smirnov test in conjunction with plots of the distribution and descriptive statistics. If normality was confirmed for the measures, parametric method (paired student t-test, Pearson's correlation test) was used. If normality was not confirmed, non-parametric method (Wilcoxon signed rank test) was used.

\section{RESULTS}

The data of 1 subject who showed sleep-onset REM period (SOREMP) on the second and the third day was excluded according to the exclusion criteria.

\section{Sleep variables derived from visual scoring}

Table 1 lists the sleep parameters derived from visual scoring compared between the nights with and without transdermal nicotine patch. Among sleep continuity measures, all parameters except TIB showed significant differences between the nights. On the nights with nicotine patch, as compared to the nights with placebo patch, TST (321.8 minutes vs. $422.7 \mathrm{~min}$ utes, $\mathrm{p}<0.01)$ and sleep efficiency $(72.5 \%$ vs. $91.6 \%, \mathrm{p}<0.01)$ were decreased and wakefulness after sleep onset (WASO) (97.8 minutes vs. 23.8 minutes, $\mathrm{p}<0.01$ ), sleep latency to stage 2 (23.7 minutes vs 9.2 minutes, $\mathrm{p}<0.01$ ), and sleep latency to REM stage (105.1 minutes vs. 74.9 minutes, $\mathrm{p}=0.05$ ) were increased. Among sleep architecture measures, on the nights with nicotine patch, as compared to the nights with placebo patch, percentage of stage 1 was increased (15.5\% vs. $10.9 \%$, $\mathrm{p}<0.01)$ and percentage of stage REM was decreased $(19.7 \%$ vs. $23.4 \%, \mathrm{p}<0.05)$. In the sleep variables for the first four consecutive cycles, non-REM sleep episode on the fourth cycle was decreased on the nights with nicotine patch compared with the placebo patch (55.4 min vs. $73.8 \mathrm{~min}, \mathrm{p}<0.05$ ) (Table 2).

\section{Sleep EEG spectral power}

EEG power density of slow wave activity in stage 2 and REM sleep of the first 3 NREM-REM cycles were plotted relative to the corresponding total values of each stage. During stage 2 sleep, SWA was significantly lower in nights with transdermal nicotine patch compared to nights with placebo patch $(\mathrm{t}=2.442, \mathrm{df}=14, \mathrm{p}<0.05)$ (Figure 1). The difference was confined to the first cycle of the nights. For spindle frequency activity, theta, alpha, beta1, and beta2 activity, no statistical differences were observed.

During REM sleep, SWA was significantly lower in nights with transdermal nicotine patch compared to nights with placebo patch on the first NREM-REM cycle of the nights $(\mathrm{t}=$ 
Table 1. Sleep variables derived from visually-scored nocturnal polysomnography after treatment with placebo or nicotine patch

\begin{tabular}{|c|c|c|c|c|c|}
\hline Variables & Placebo & Nicotine & $\mathrm{T}$ or $\mathrm{Z}$ & $\mathrm{df}$ & $\mathrm{p}$ \\
\hline $\mathrm{TIB}$ (min) & $461.5(37.9)^{*}$ & $447.3(45.7)$ & 1.280 & 14 & 0.221 \\
\hline TST (min) & $422.7(48.7)$ & $321.8(96.0)$ & 4.323 & 14 & $0.001^{\dagger}$ \\
\hline SE (\%) & $91.6(7.4)$ & $72.5(21.2)$ & 4.012 & 14 & $0.001^{\dagger}$ \\
\hline Latency to stage $1(\mathrm{~min})$ & $4.6(6.8)$ & $17.5(37.0)$ & $-2.329 \ddagger$ & 14 & $0.003^{\dagger}$ \\
\hline Latency to stage $2(\mathrm{~min})$ & $9.2(7.6)$ & $23.7(35.8)$ & $-2.095^{\ddagger}$ & 14 & $0.007^{\dagger}$ \\
\hline Latency to REM sleep (min) & $74.9(37.1)$ & $105.1(63.7)$ & -2.146 & 14 & $0.050^{\dagger}$ \\
\hline WASO (min) & $23.8(36.2)$ & $97.8(105.9)$ & $-2.819^{\ddagger}$ & 14 & $0.008^{\dagger}$ \\
\hline Stage $1(\%)$ & $10.9(5.5)$ & $15.5(7.7)$ & -3.204 & 14 & $0.006^{\dagger}$ \\
\hline Stage $2(\%)$ & $61.0(5.4)$ & $58.0(10.9)$ & 1.292 & 14 & 0.217 \\
\hline Stage $3(\%)$ & $5.5(4.7)$ & $6.3(5.3)$ & -1.260 & 14 & 0.228 \\
\hline Stage $4(\%)$ & $0.5(1.1)$ & $2.4(5.4)$ & $-1.648^{\ddagger}$ & 14 & 0.063 \\
\hline REM stage (\%) & $23.4(3.2)$ & $19.7(4.8)$ & 2.463 & 14 & $0.027^{\dagger}$ \\
\hline SWS (\%) & $6.0(5.4)$ & $8.7(9.7)$ & -1.721 & 14 & 0.107 \\
\hline
\end{tabular}

${ }^{*}$ mean (standard deviation) is shown, ${ }^{\dagger} \mathrm{p}<0.05,{ }^{\ddagger} \mathrm{z}$-value (by Wilcoxon signed ranks test). TIB: time in bed, TST: total sleep time, SE: sleep efficiency, REM: rapid eye movement, WASO: wake after sleep onset, SWS, slow wave sleep

Table 2. Sleep variables derived from visually-scored nocturnal polysomnography after treatment with placebo or nicotine patch for the first four consecutive cycles

\begin{tabular}{|c|c|c|c|c|c|}
\hline Variables & Placebo & Nicotine & $\mathrm{T}$ or $\mathrm{Z}$ & $\mathrm{df}$ & $\mathrm{p}$ \\
\hline \multicolumn{6}{|l|}{ Cycle 1 (min) } \\
\hline NREM sleep episode & $80.0(30.9)^{*}$ & $133.2(100.7)$ & -2.130 & 14 & 0.051 \\
\hline REM sleep episode & $17.6(8.0)$ & $23.4(11.3)$ & -1.682 & 14 & 0.115 \\
\hline SWS & $12.9(15.6)$ & $16.1(14.9)$ & -0.911 & 14 & 0.378 \\
\hline \multicolumn{6}{|l|}{ Cycle 2 (min) } \\
\hline NREM sleep episode & $84.2(33.8)$ & $96.3(56.3)$ & -0.618 & 14 & 0.547 \\
\hline REM sleep episode & $25.5(16.2)$ & $21.0(12.3)$ & 0.801 & 13 & 0.438 \\
\hline SWS & $8.7(11.7)$ & $6.0(10.0)$ & $-0.578^{\ddagger}$ & 14 & 0.563 \\
\hline \multicolumn{6}{|l|}{ Cycle 3 (min) } \\
\hline NREM sleep episode & $72.2(12.5)$ & $94.3(39.5)$ & -2.141 & 13 & 0.052 \\
\hline REM sleep episode & $27.5(17.1)$ & $25.3(20.1)$ & 0.392 & 8 & 0.705 \\
\hline SWS & $1.8(2.7)$ & $1.9(5.0)$ & $-0.475^{\ddagger}$ & 13 & 0.635 \\
\hline \multicolumn{6}{|l|}{ Cycle 4 (min) } \\
\hline NREM sleep episode & $73.8(15.7)$ & $55.4(20.7)$ & 2.769 & 8 & $0.024^{\dagger}$ \\
\hline REM sleep episode & $31.0(16.6)$ & $10.7(4.7)$ & 1.231 & 2 & 0.343 \\
\hline SWS & $1.2(3.2)$ & $0.17(0.4)$ & $-0.680^{\ddagger}$ & 8 & 0.496 \\
\hline
\end{tabular}

${ }^{*}$ mean (standard deviation) is shown, ${ }^{\dagger} \mathrm{p}<0.05,{ }^{\ddagger} \mathrm{z}$-value (by Wilcoxon signed ranks test). NREM: non-rapid eye movement, REM: rapid eye movement, SWS: slow wave sleep

2.498, $\mathrm{df}=14, \mathrm{p}<0.05$ ) (Figure 2). During REM sleep, alpha activity was significantly higher in nights with transdermal nicotine patch compared to nights with placebo patch also on the first NREM-REM cycle of the nights $(\mathrm{t}=-2.673, \mathrm{df}=14, \mathrm{p}<$ 0.05 ) (Figure 3). For spindle frequency activity, theta, beta1, and beta 2 activity, no statistical differences were observed.

\section{Heart rate variability}

LF and HF power, LHR, VLF, R-R means, and R-R variance in stage 2 and stage REM sleep were compared between the nights with transdermal nicotine patch and placebo patch. However, no statistical differences were observed.

LHR, indicating sympathetic nervous system activity, was $12.7 \pm 31.2$ during REM sleep in the nights with transdermal nicotine patch but not significantly higher than the nights with placebo patch $(8.3 \pm 15.8)$. We examined the correlation between LHR and other variables including TST, sleep efficiency, WASO, and SWA. Significant correlation was found 


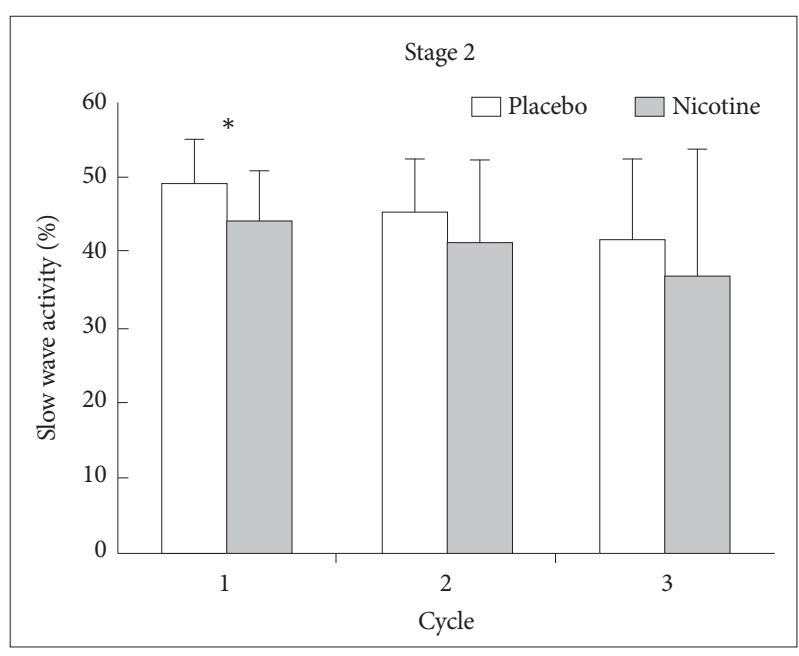

Figure 1. EEG power density of slow wave activity in stage 2 sleep of the first 3 NREM-REM sleep cycles. ${ }^{*} p<0.05$ by paired t-test. EEG: electroencephalogram, NREM: non-rapid eye movement, REM: rapid eye movement.

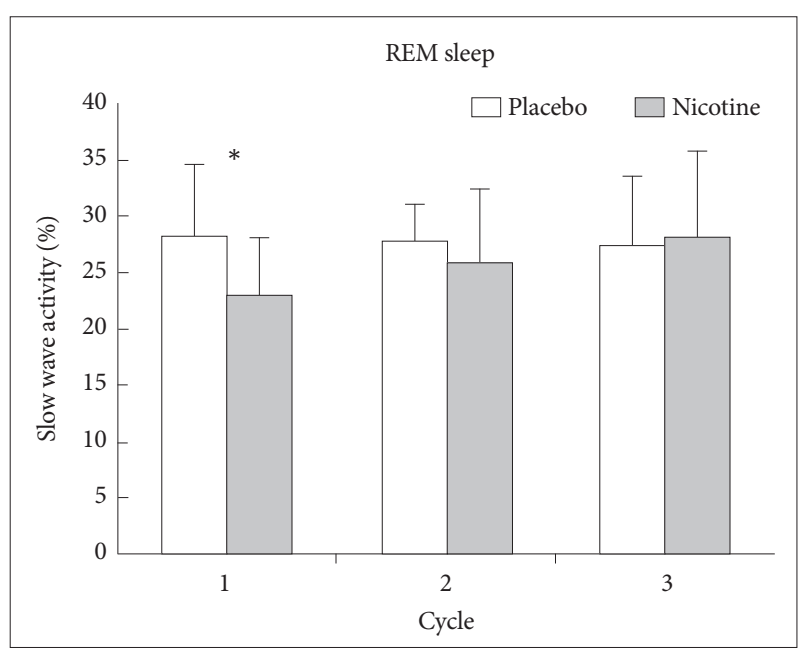

Figure 2. EEG power density of slow wave activity in REM sleep of the first 3 NREM-REM sleep cycles. ${ }^{*} p<0.05$ by paired t-test. EEG: electroencephalogram, NREM: non-rapid eye movement, REM: rapid eye movement.

between LHR and WASO during stage 2 sleep in the nights with transdermal nicotine patch $(\mathrm{r}=0.535, \mathrm{p}<0.05)$ (Figure 4$)$, but not in the nights with placebo patch. A tendency for reverse correlation between LHR and SWA during stage 2 sleep in the nights with transdermal nicotine patch $(\mathrm{r}=-0.442, \mathrm{p}=$ 0.099) was observed.

\section{DISCUSSION}

As we predicted, the current study shows that transdermal nicotine patch causes significant effects on sleep continuity, sleep architecture, sleep EEG and sleep EKG spectral parameters. Based on parameters derived from visual scoring, the effects of nicotine on sleep were mostly characterized by in-

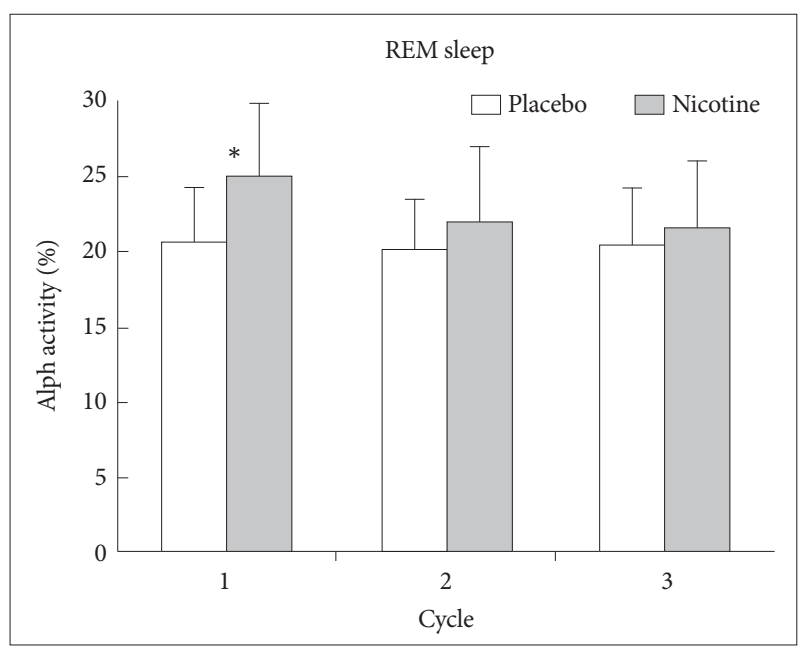

Figure 3. EEG power density of alpha activity in REM sleep of the first 3 NREM-REM sleep cycles. ${ }^{*} p<0.05$ by paired t-test. EEG: electroencephalogram, NREM: non-rapid eye movement, REM: rapid eye movement.

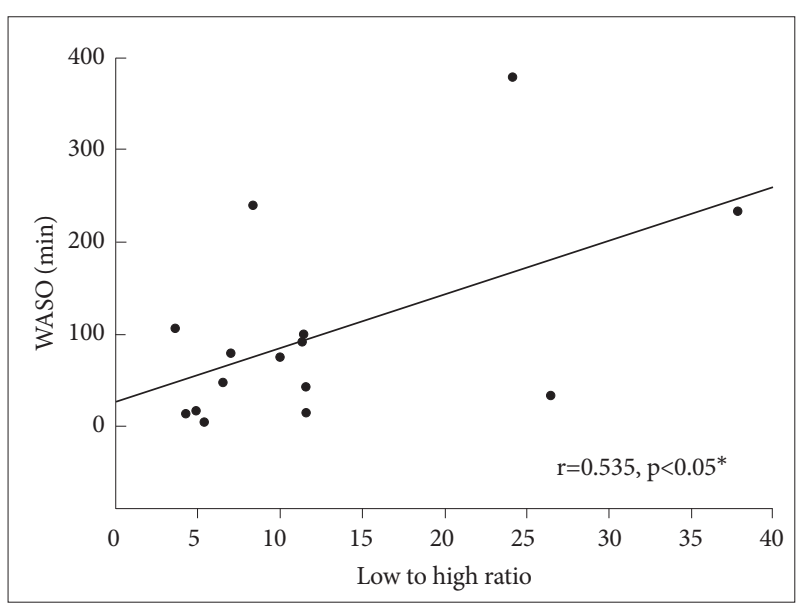

Figure 4. Correlation between wake after sleep onset (WASO) and low to high ratio (LHR) in stage 2 after administration of nicotine patch. *Pearson's correlation.

creased wakefulness and decreased TST and sleep efficiency. Moreover, a clear trend toward REM sleep reduction in the nights with transdermal nicotine patch was observed. These findings are consistent with prior studies. ${ }^{3,4}$

Nicotine is known to act as a presynaptic agonist to release acetylcholine from the nerve terminal. ${ }^{32}$ Acetylcholine has a key role in inducing and maintaining REM sleep. ${ }^{33}$ Therefore, it may be expected that nicotine would enhance REM sleep. However, in most of studies with non-smoking normal volunteers, REM sleep was reported to be reduced by transdermal nicotine as in our results. Only Salin-Pascual and Drucker-Colin ${ }^{34}$ reported an increase in REM sleep in both normal volunteers and depressed patients who were chronically treated with nicotine patches. They administered nicotine patch to subjects for 4 days consecutively, and found increase in REM sleep, with the exception of on the first day. Thus, 
whether decreases in REM sleep are limited to the acute phase of nicotine patch needs to be further clarified.

Whereas visual scoring of sleep failed to detect significant modifications in the depth of sleep, spectral analysis revealed a number of changes. Compared to the nights with placebo patch, nights with nicotine patch had significantly less SWA in the stage 2 and REM sleep of the first NREM and REM sleep cycles, respectively. We can infer nicotine suppressed homeostatic sleep propensity and disturbed deep sleep, as SWA represents a physiological marker of the homeostatic facet of sleep regulation. ${ }^{11-13}$ Because SWA generally declines across successive NREMS episodes, the nicotine effects on slow wave sleep is expected to be most prominent in the first NREM cycle.

In the first REM sleep episode, alpha activity was significantly higher in the nights with nicotine patch. Endo et al. ${ }^{35}$ reported that alpha activity is reversely correlated with REM sleep propensity and alpha activity is regarded as a physiological marker for REM sleep homeostasis. ${ }^{36}$ Therefore, increased alpha activity in REM sleep in the nights with nicotine patch means can be interpreted as suppression of REM sleep propensity by nicotine.

In the current study, we could not find significant difference in sympathetic activation, measured by EKG frequency power. However, the significant correlation between LHR and WASO and the trend for reverse correlation between LHR and SWA during stage 2 sleep in the nights with transdermal nicotine patch suggests the role of sympathetic activation in promoting arousal and suppressing homeostatic sleep propensity. This is probably due to the catecholamine stimulating effects of nicotine which produces a number of physiological effects including increased heart rate and blood pressure and dose dependent increase in the secretion of prolactine and ACTH, resulting in a subsequent increase in corticosteroid secretion..$^{20,22,37}$

Our results cannot be generalized to regular smokers. The nicotine blood levels in the nights with transdermal nicotine would have increased during sleep, whereas in regular smokers, is likely to be decreased during sleep. Also, both pharmacokinetic and pharmacodynamic effects of transdermal nicotine can differ between smokers and non-smokers. Our study was not aimed to find the change of sleep architecture in smokers, so these were not the limitations of this study. Rather, our results suggest that beginning smokers can suffer from sleep difficulty. One of the limitations of this study is small sample size.

The use of transdermal nicotine patch in smoking cessation may be an effective treatment strategy. Wetter et al. ${ }^{38}$ suggested that among dependent smokers tobacco withdrawal increased objectively assessed sleep disturbance and that nicotine replacement resulted in postcessation improvements in impor- tant polysomnographic measures of sleep quality. However, a meta-analysis result showed that sleep disturbance caused by cigarette withdrawal does not seem to ameliorate with transdermal nicotine patch and paradoxically may even be increased. ${ }^{39}$ Our study results demonstrated that transdermal nicotine patch significantly disrupted sleep continuity, sleep architecture, and homeostatic sleep propensity.

\section{REFERENCES}

1. Roehrs T, Roth T. Medication and Substance Abuse. In: Kryger M, Roth T, Dement W, Editors. Principles and Practice of Sleep Medicine. WC, Philadelphia: Elsevier Saunders, 2011, p.1512-1523.

2. Soldatos CR, Kales JD, Scharf MB, Bixler EO, Kales A. Cigarette smoking associated with sleep difficulty. Science 1980;207:551-553.

3. Davila DG, Hurt RD, Offord KP, Harris CD, Shepard JW Jr. Acute effects of transdermal nicotine on sleep architecture, snoring, and sleepdisordered breathing in nonsmokers. Am J Respir Crit Care Med 1994; 150:469-474.

4. Gillin JC, Lardon M, Ruiz C, Golshan S, Salin-Pascual R. Dose-dependent effects of transdermal nicotine on early morning awakening and rapid eye movement sleep time in nonsmoking normal volunteers. J Clin Psychopharmacol 1994;14:264-267.

5. Salin-Pascual RJ, de la Fuente JR, Galicia-Polo L, Drucker-Colin R. Effects of transdermal nicotine on mood and sleep in nonsmoking major depressed patients. Psychopharmacology (Berl) 1995;121:476-479.

6. Zhang L, Samet J, Caffo B, Punjabi NM. Cigarette smoking and nocturnal sleep architecture. Am J Epidemiol 2006;164:529-537.

7. Jaehne A, Unbehaun T, Feige B, Lutz UC, Batra A, Riemann D. How smoking affects sleep: a polysomnographical analysis. Sleep Med 2012; 13:1286-1292.

8. Borbely AA. New techniques for the analysis of the human sleep-wake cycle. Brain Dev 1986;8:482-488.

9. Achermann P, Borbely AA. Sleep Homeostasis and Models of Sleep Regulation. In: Kryger M, Roth T, Dement W, Editors. Principles and Practice of Sleep Medicine. WC, Philadelphia: Elsevier Saunders, 2011, p.431-444.

10. Zhang L, Samet J, Caffo B, Bankman I, Punjabi NM. Power spectral analysis of EEG activity during sleep in cigarette smokers. Chest 2008; 133:427-432.

11. Borbely AA, Baumann F, Brandeis D, Strauch I, Lehmann D. Sleep deprivation: effect on sleep stages and EEG power density in man. Electroencephalogr Clin Neurophysiol 1981;51:483-495.

12. Finelli LA, Baumann H, Borbely AA, Achermann P. Dual electroencephalogram markers of human sleep homeostasis: correlation between theta activity in waking and slow-wave activity in sleep. Neuroscience 2000;101:523-529.

13. Rodriguez AV, Funk CM, Byazovskiy VV, Nir Y, Tononi G, Cirelli C. Why does sleep slow-wave activity increase after extended wake? Assessing the effects of increased cortical firing during wake and sleep. J Neurosci 2016;36:12436-12447.

14. Dijk DJ, Hayes B, Czeisler CA. Dynamics of electroencephalographic sleep spindles and slow wave activity in men: effect of sleep deprivation. Brain Res 1993;626:190-199.

15. Aeschbach D, Cajochen C, Landolt H, Borbely AA. Homeostatic sleep regulation in habitual short sleepers and long sleepers. Am J Physiol 1996;270:R41-R53.

16. Campbell IG, Higgins LM, Darchia N, Feinberg I. Homeostatic behavior of fast fourier transform power in very low frequency non-rapid eye movement human electroencephalogram. Neuroscience 2006;140: 1395-1399.

17. Bersagliere A, Achermann P. Slow oscillations in human non-rapid eye movement sleep electroencephalogram: effects of increased sleep 
pressure. J Sleep Res 2010;19:228-237.

18. Tononi G, Cirelli C. Sleep and synaptic homeostasis: a hypothesis. Brain Res Bull 2003;62:143-150

19. Stein PK, Bosner MS, Kleiger RE, Conger BM. Heart rate variability: a measure of cardiac autonomic tone. Am Heart J 1994;127:1376-1381.

20. Newhouse PA, Sunderland T, Narang PK, Mellow AM, Fertig JB, Lawlor BA, et al. Neuroendocrine, physiologic, and behavioral responses following intravenous nicotine in nonsmoking healthy volunteers and in patients with alzheimer's disease. Psychoneuroendocrinology 1990;15: 471-484.

21. Jones GM, Sahakian BJ, Levy R, Warburton DM, Gray JA. Effects of acute subcutaneous nicotine on attention, information processing and short-term memory in alzheimer's disease. Psychopharmacology (Berl) 1992;108:485-494.

22. Benowitz NL. Pharmacology of nicotine: Addiction and therapeutics. Annu Rev Pharmacol Toxicol 1996;36:597-613.

23. Parati G, Saul JP, Di Rienzo M, Mancia G. Spectral analysis of blood pressure and heart rate variability in evaluating cardiovascular regulation. A critical appraisal. Hypertension 1995;25:1276-1286.

24. Lucini D, Bertocchi F, Malliani A, Pagani M. A controlled study of the autonomic changes produced by habitual cigarette smoking in healthy subjects. Cardiovasc Res 1996;31:633-639.

25. Stein PK, Rottman JN, Kleiger RE. Effect of $21 \mathrm{mg}$ transdermal nicotine patches and smoking cessation on heart rate variability. Am J Cardiol 1996;77:701-705.

26. Lucini D, Bertocchi F, Malliani A, Pagani M. Autonomic effects of nicotine patch administration in habitual cigarette smokers: a doubleblind, placebo-controlled study using spectral analysis of RR interval and systolic arterial pressure variabilities. J Cardiovasc Pharmacol 1998; 31:714-720

27. Hamilton M. A rating scale for depression. J Neurol Neurosurg Psychiatry 1960;23:56-62.

28. Beck AT, Ward CH, Mendelson M, Mock J, Erbaugh J. An inventory for measuring depression. Arch Gen Psychiatry 1961;4:53-63.

29. Jasper HH (committee chairman). The ten twenty electrode system of the International Federation. Electroencephalogr Clin Neurophysiol 1958;10:371-375.

30. Rechtschaffen A, Kales A. A Manual of Standardized Terminology, Technique, and Scoring System for Sleep Stages of Human Subjects. Los Angeles: BIS/BRI, University of California; 1968.

31. Feinberg I, Floyd TC. Systematic trends across the night in human sleep cycles. Psychophysiology 1979;16:283-291.

32. De Sarno P, Giacobini E. Modulation of acetylcholine release by nicotinic receptors in the rat brain. J Neurosci Res 1989;22:194-200.

33. Siegel J. Rem sleep. In: Kryger M, Roth T, Dement W, Editors. Principles and practice of sleep medicine. WC, Philadelphia: Elsevier Saunders, 2011, p.92-111.

34. Salin-Pascual RJ, Drucker-Colin R. A novel effect of nicotine on mood and sleep in major depression. Neuroreport 1998;9:57-60.

35. Endo T, Roth C, Landolt HP, Werth E, Aeschbach D, Achermann P, et al. Selective rem sleep deprivation in humans: Effects on sleep and sleep eeg. Am J Physiol 1998;274:R1186-R1194.

36. Roth C, Achermann P, Borbely AA. Alpha activity in the human rem sleep EEG: Topography and effect of REM sleep deprivation. Clin Neurophysiol 1999;110:632-635.

37. Fu Y, Matta SG, Brower VG, Sharp BM. Norepinephrine secretion in the hypothalamic paraventricular nucleus of rats during unlimited access to self-administered nicotine: An in vivo microdialysis study. J Neurosci 2001;21:8979-8989.

38. Wetter DW, Fiore MC, Baker TB, Young TB. Tobacco withdrawal and nicotine replacement influence objective measures of sleep. J Consult Clin Psychol 1995;63:658-667.

39. Greenland S, Satterfield MH, Lanes SF. A meta-analysis to assess the incidence of adverse effects associated with the transdermal nicotine patch. Drug Saf 1998;18:297-308. 